Pacific Journal of Mathematics

NEVANLINNA PARAMETRIZATION FOR THE EXTENDED 


\section{NEVANLINNA PARAMETRIZATIONS FOR THE EXTENDED INTERPOLATION PROBLEM}

\section{SEchiko TAKaHASHI}

Let $\mathscr{B}$ be the set of holomorphic functions $f$ with $|f| \leq 1$ in the open unit disc $D=\{z \in \mathbb{C}:|z|<1\}$. Let $\sigma=\left\{z_{1}, z_{2}, \ldots\right\}$ be a finite or infinite sequence of distinct points in $D$ and, for each point $z_{i} \in \sigma,\left(c_{i 0}, \ldots, c_{i n_{t}-1}\right)$ be an ordered $n_{i}$-tuple of complex numbers $\left(0<n_{i}<+\infty\right)$. The problem is to find a function $f$ which belongs to $\mathscr{B}$ and satisfies the extended interpolation conditions

$$
f(z)=\sum_{\alpha=0}^{n_{i}-1} c_{i \alpha}\left(z-z_{i}\right)^{\alpha}+\mathbf{O}\left(\left(z-z_{i}\right)^{n_{l}}\right) \quad\left(\forall z_{l} \in \sigma\right) .
$$

Let $\mathscr{E}$ denote the set of all solutions of this problem (EI) in $\mathscr{B}$ and assume the hypothesis

$$
\mathscr{E} \text { has at least two elements. }
$$

A bijection $\pi: \mathscr{B} \rightarrow \mathscr{E}$ is called Nevanlinna parametrization of $\mathscr{E}$ if there exist four functions $P, Q, R$, and $S$ holomorphic in $D$ and such that $R g+S \not \equiv 0, \pi(g)=(P g+Q) /(R g+S)$ for any $g \in \mathscr{B}$. The existence, some properties and some applications of such parametrizations are shown. One has a bijection between the set of Nevanlinna parametrizations of $\mathscr{E}$ and the group of Möbius transformations.

In our previous paper [24], $\sigma=\left\{z_{1}, \ldots, z_{k}\right\}$ being finite, we constructed in a simple manner an Hermitian $n \times n$ matrix $A$ from the given data $\left\{z_{i}\right\}$ and $\left\{c_{i \alpha}\right\} \quad\left(1 \leq i \leq k, 0 \leq \alpha \leq n_{i}-1\right)$, where $n=\sum_{i=1}^{k} n_{i}$, and established the following theorems which unify the coefficient theorem of Carathéodory-Toeplitz-Schur [3], [26], and [19] and the interpolation theorem of Pick [17]:

THEOREM E. The problem (EI) admits a solution in $\mathscr{B}$ if and only if $A \geq 0$ (positive semidefinite). If so, among the solutions, there is a finite Blaschke product of degree $\leq n$.

THEOREM U. The solution of the problem (EI) in $\mathscr{B}$ is unique if and only if $A \geq 0$ and $\operatorname{det} A=0$. In this case, the unique solution is a finite Blaschke product whose degree is equal to the rank of $A$. 
Conversely, if a finite Blaschke product of degree $<n$ is a solution of (EI) in $\mathscr{B}$, then it is the unique solution of (EI) in $\mathscr{B}$.

In the case where $\sigma$ is infinite, we shall treat briefly the existence of solutions and Denjoy's uniqueness theorem in $\S 7$ below.

Now, we proceed to the case in which the problem (EI) has more than one solution in $\mathscr{B}$. As we said above, we shall assume, always in this paper except in $\S 7$, the hypothesis $(\mathrm{H})$. Nevanlinna parametrizations are very powerful for our study of the present case.

In $\S 5$, we shall prove that, under the hypothesis $(\mathrm{H})$, there exists a Nevanlinna parametrization of $\mathscr{E}$, somewhat more axiomatically than Nevanlinna [13] did in the classical case where all $n_{i}$ reduce to 1 , that is, only the values at $z_{i}$ are prescribed.

After having studied some properties of Nevanlinna parametrizations in $\S 3$, we shall proceed in $\S 4$ to consider the totality $\mathscr{P}$ of Nevanlinna parametrizations of $\mathscr{E}$. In [13], Nevanlinna introduced some parameters in each step of his algorithm and then specialized them conveniently for his purpose. It is natural to ask how many parameters are essentially efficient to induce different parametrizations. Our answer is as follows:

The group $G$ of Möbius transformations

$$
\tau(z)=\lambda \frac{z+a}{1+\bar{a} z} \quad(|\lambda|=1,|a|<1),
$$

here regarded as analytic automorphisms of the closed unit disc $\bar{D}=$ $D \cup \partial D$, operates canonically on $\mathscr{P}$ in such a way that

$$
\left(\tau^{*}(\pi)\right)(g)=\pi(\tau \circ g) \quad(\pi \in \mathscr{P}, g \in \mathscr{B}) .
$$

We shall prove in $\S 4$ that, under the hypothesis $(\mathrm{H})$, for any $\pi_{0}, \pi \in$ $\mathscr{P}$, there exists one and only one $\tau \in G$ such that $\pi=\tau^{*}\left(\pi_{0}\right)$. Thus, with $\pi_{0} \in \mathscr{P}$ fixed, $\mathscr{P}$ and $G$ correspond bijectively.

Finally, we want to mention that the existence theorem of Nevanlinna parametrizations in $\S 5$ and some parts of the results in $\S 6$ and $\S 7$ are reported in [25].

2. Preliminaries. Schur's triangular matrices will make easy and transparent our calculations of coefficients in the sequel. To a function

$$
f(z)=\sum_{\alpha=0}^{\infty} c_{\alpha}\left(z-z_{0}\right)^{\alpha}
$$


holomorphic at $z_{0}$ and to a positive integer $m$, we associate the following $m \times m$ triangular matrix

$$
\Delta\left(f ; z_{0} ; m\right)=\left(\begin{array}{cccc}
c_{0} & & & \\
c_{1} & c_{0} & & \\
\vdots & \ddots & \ddots & \\
c_{m-1} & \cdots & c_{1} & c_{0}
\end{array}\right)
$$

For each $z_{i} \in \sigma$, replacing $m$ by $n_{i}$ and $c_{\alpha}$ by $c_{i \alpha} \quad(0 \leq \alpha \leq$ $\left.n_{i}-1\right)$, we obtain an $n_{i} \times n_{i}$ triangular matrix $C_{i}$. Then we may say that for an $f \in \mathscr{B}$ to belong to $\mathscr{E}$ it is necessary and sufficient that $\Delta\left(f ; z_{i} ; n_{i}\right)=C_{i}$ for any $z_{i} \in \sigma$. One sees immediately

Proposition 1. Let $f$ and $g$ be holomorphic at $z_{0}$. Then we have

(a) $\Delta\left(f+g ; z_{0} ; m\right)=\Delta\left(f ; z_{0} ; m\right)+\Delta\left(g ; z_{0} ; m\right)$;

(b) $\Delta\left(f g ; z_{0} ; m\right)=\Delta\left(f ; z_{0} ; m\right) \cdot \Delta\left(g ; z_{0} ; m\right)=\Delta\left(g ; z_{0} ; m\right)$. $\Delta\left(f ; z_{0} ; m\right)$;

(c) $\Delta\left(1 ; z_{0} ; m\right)=I_{m}$ (the unit matrix of order $\left.m\right)$.

Next, to our extended interpolation problem (EI), we assign the Blaschke product

$$
B(z)=\prod_{z_{l} \in \sigma}\left(\lambda_{i} \frac{z-z_{i}}{1-\bar{z}_{i} z}\right)^{n_{\imath}},
$$

where $\lambda_{i}=1$ if $z_{i}=0$; and $\lambda_{i}=-\left|z_{i}\right| / z_{i}$ if $z_{i} \neq 0$. We point out here that, when $\sigma$ is infinite, the hypothesis $(\mathrm{H})$ implies the convergence of this infinite product. In fact, if $f_{1}, f_{2} \in \mathscr{E}$ and $f_{1} \neq \equiv f_{2}$, then $f_{1}-f_{2}$ is bounded and $\not \equiv 0$ (does not vanish identically). Each $z_{i} \in \sigma$ is its zero of order $\geq n_{i}$, so that $\sum_{z_{i} \in \sigma} n_{i}\left(1-\left|z_{i}\right|\right)<+\infty$ and the infinite product converges in $D$. Thus, $\sigma$ being finite or infinite, $B$ is holomorphic in $D$ and vanishes exactly on $\sigma$ with the order $n_{i}$ at $z_{i} \in \sigma$.

We recall the following well-known properties of linear transformations.

Proposition 2. Let

$$
\tau(z)=\frac{p z+q}{r z+s} \quad(p, q, r, s \in \mathbb{C} ; p s-q r \neq 0)
$$

be a linear transformation. If $\tau(D)$ is bounded, then $|r|<|s|$ and $\tau(D)$ is the open disc of center $(q \bar{s}-p \bar{r}) /\left(|s|^{2}-|r|^{2}\right)$ and of radius 
$|p s-q r| /\left(|s|^{2}-|r|^{2}\right)$. If $\tau(D) \subset D$ then $|p| \leq|s|,|q|<|s|,|r|<|s|$. If $\tau(\bar{D}) \subset D$ then $|p|<|s|$.

3. Basic properties of Nevanlinna parametrizations. Until $\S 7$, we consider always the extended interpolation problem (EI) under the hypothesis $(\mathrm{H})$. For each $z_{0} \in D$, let

$$
W\left(z_{0}\right)=\left\{f\left(z_{0}\right): f \in \mathscr{E}\right\}
$$

denote the set of values taken at $z_{0}$ by all solutions of $(\mathrm{EI})$ in $\mathscr{B}$. We have $W\left(z_{0}\right) \subset D$. In fact, $W\left(z_{0}\right) \subset \bar{D}$ is evident. If there were an $f \in \mathscr{E}$ such that $f\left(z_{0}\right) \in \partial D$, then $f$ is constant by the maximum principle, so that $\left|c_{10}\right|=1$. All solutions of $(\mathrm{EI})$ in $\mathscr{B}$ reduce to the constant $c_{10}$, contrary to $(\mathrm{H})$. For any $z_{i} \in \sigma, W\left(z_{i}\right)$ reduces to a point: $W\left(z_{i}\right)=\left\{c_{i 0}\right\}$.

As we defined in the Introduction, a Nevanlinna parametrization of $\mathscr{E}$ is a bijective mapping $\pi: \mathscr{B} \rightarrow \mathscr{E}$ such that there exist four holomorphic functions $P, Q, R$, and $S$ in $D$ satisfying

$$
\pi(g)=\frac{P g+Q}{R g+S}, \quad R g+S \not \equiv 0 \quad(\forall g \in \mathscr{B}) .
$$

We shall say that the quadruple $(P, Q, R, S)$ represents $\pi$. To this $\pi$ and to each $z_{0} \in D$, we associate the mapping $\pi_{z_{0}}: \bar{D} \rightarrow W\left(z_{0}\right)$ defined by

$$
\pi_{z_{0}}(\zeta)=\pi(\zeta)\left(z_{0}\right) \quad(\zeta \in \bar{D}),
$$

where each $\zeta \in \bar{D}$ is regarded as a constant function. If $P\left(z_{0}\right) S\left(z_{0}\right)-$ $Q\left(z_{0}\right) R\left(z_{0}\right) \neq 0$, then we have

$$
\pi_{z_{0}}(\zeta)=\frac{P\left(z_{0}\right) \zeta+Q\left(z_{0}\right)}{R\left(z_{0}\right) \zeta+S\left(z_{0}\right)} \quad(\forall \zeta \in \bar{D})
$$

and, taking account of constant functions, we see that $\pi_{z_{0}}: \bar{D} \rightarrow$ $W\left(z_{0}\right)$ is bijective and hence $W\left(z_{0}\right)$ is a nondegenerate closed disc in $D$.

Using Proposition 2, we observe now some basic properties of quadruples representing Nevanlinna parametrizations.

Propositions 3. Let $(P, Q, R, S)$ represent Nevanlinna parametrization of $\mathscr{E}$. Then we have

(a) $S \not \equiv 0$ in $D$

(b) $P S-Q R \not \equiv 0$;

(c) $P / S, Q / S$, and $R / S$ are all holomorphic in $D$; 
(d) $|P / S|<1,|Q / S|<1$, and $|R / S|<1$ in $D$;

(e) $Q / S \in \mathscr{E}$.

Proof. Taking $g \equiv 0$, one sees $S \not \equiv 0$ and $Q / S \in \mathscr{E}$. If $P S-Q R \equiv$ 0 , the bijection $\pi$ would be a constant mapping, which is impossible. To see (c) and (d), take $z_{0} \in D \backslash \sigma$ such that $S\left(z_{0}\right) \neq 0$ and $P\left(z_{0}\right) S\left(z_{0}\right)-Q\left(z_{0}\right) R\left(z_{0}\right) \neq 0$. Then, since $W\left(z_{0}\right) \subset D$, Proposition 2 shows that the inequalities in (d) hold at $z_{0}$ and hence in $D$ with the exception of a discrete set. Riemann's theorem of removable singularities yields (c) and the maximum principle implies (d).

By virtue of this proposition, for any representing quadruple $(P, Q, R, S)$, we shall assume always $S \neq 0$ in $D$ ( $S$ has no zeros in $D$ ) and sometimes $S \equiv 1$. Notice that by this assumption we have $R g+S \neq 0$ in $D$ for any $g \in \mathscr{B}$. Moreover, for any $z_{0} \in D$, $\pi_{z_{0}}$ is surjective and we have the relation $(*)$.

4. Totality of Nevanlinna parametrizations. As in the Introduction, let $\mathscr{P}$ denote the set of Nevanlinna parametrizations of $\mathscr{E}$ and $G$ the group of Möbius transformations. For $\tau \in G$ and $\pi \in \mathscr{P}$, we have by definition

$$
\left(\tau^{*}(\pi)\right)(g)=\pi(\tau \circ g) \quad(g \in \mathscr{B}),
$$

and, for $\tau_{1}, \tau_{2} \in G$,

$$
\left(\tau_{1} \circ \tau_{2}\right)^{*}=\tau_{2}^{*} \circ \tau_{1}^{*} \quad \text { and } \quad i d^{*}=i d .
$$

Thus $G$ operates on $\mathscr{P}$.

In the next $\S 5, \mathscr{P} \neq \varnothing$ will be shown. In this section, we shall prove that there is a bijective correspondence between $\mathscr{P}$ and $G$, provided that $\mathscr{P} \neq \varnothing$. For this purpose, we need two lemmas.

Lemma 1. Let $z_{0} \in D, w_{0} \in \partial W\left(z_{0}\right)$, and $\pi \in \mathscr{P}$. Assume that $W\left(z_{0}\right)$ does not reduce to a point. Then there is a unique $f \in \mathscr{B}$ such that $f\left(z_{0}\right)=w_{0}$. This unique function $f$ is of the form $f=\pi(\zeta)$ $(\zeta \in \partial D)$.

In fact, as $W\left(z_{0}\right)$ is closed, there is an $f \in \mathscr{E}$ such that $f\left(z_{0}\right)=w_{0}$. Take $g \in \mathscr{B}$ with $\pi(g)=f$. Since $\pi_{z_{0}}\left(g\left(z_{0}\right)\right)=f\left(z_{0}\right) \in \partial W\left(z_{0}\right)$, $g\left(z_{0}\right) \in \partial D$ and $g$ reduces to a constant $\zeta$ with $\pi_{z_{0}}(\zeta)=w_{0}$. The uniqueness is trivial.

Lemma 2. Let $z_{0} \in D$ and $\pi_{1}, \pi_{2} \in \mathscr{P}$. If $W\left(z_{0}\right)$ does not reduce to a point and if $\pi_{1 z_{0}}=\pi_{2 z_{0}}$ then we have $\pi_{1}=\pi_{2}$. 
To see this, let $\pi_{\nu}(\nu=1,2)$ be represented by $\left(P_{\nu}, Q_{\nu}, R_{\nu}, 1\right)$ and put

$$
F_{\nu}(z, \zeta)=\frac{P_{\nu}(z) \zeta+Q_{\nu}(z)}{R_{\nu}(z) \zeta+1} \quad((z, \zeta) \in D \times \bar{D}) .
$$

Note that $F_{1}$ and $F_{2}$ are holomorphic on $D \times \bar{D}$ and that, for any fixed $\zeta \in \bar{D}$, they belong to $\mathscr{E}$ as functions of $z$. By assumption, $F_{1}=F_{2}$ on $\left\{z_{0}\right\} \times \bar{D}$. By Lemma $1, F_{1}=F_{2}$ on $D \times \partial D$ and hence on $D \times \bar{D}$. Therefore, for any $g \in \mathscr{B}$, we have $F_{1}(z, g(z))=F_{2}(z, g(z))$, which shows $\pi_{1}=\pi_{2}$.

Theorem 1. Assume the hypothesis $(\mathrm{H})$ and let $\pi, \pi_{0} \in \mathscr{P}$. Then there exists one and only one $\tau \in G$ such that $\pi=\tau^{*}\left(\pi_{0}\right)$.

Proof. Take one $z_{0} \in D$ such that $W\left(z_{0}\right)$ does not reduce to a point. There exists one and only one $\tau \in G$ such that $\pi_{z_{0}}=\pi_{0 z_{0}} \circ$ $\tau$. Since $\pi_{0 z_{0}} \circ \tau=\left(\tau^{*}\left(\pi_{0}\right)\right)_{z_{0}}$, Lemma 2 shows $\pi=\tau^{*}\left(\pi_{0}\right)$. The uniqueness is now clear.

Note that if we fix one $\pi_{0} \in \mathscr{P}$ as a base point, $\tau \mapsto \tau^{*}\left(\pi_{0}\right)$ gives a bijective mapping of $G$ onto $\mathscr{P}$, so that the totality $\mathscr{P}$ of Nevanlinna parametrizations has only three real parameters.

5. Existence of Nevanlinna parametrizations. By means of SchurNevanlinna's algorithm, let us establish the existence theorem of parametrizations.

THEOREM 2. For the extended interpolation problem (EI) and under the hypothesis $(\mathrm{H})$, there exists a Nevanlinna parametrization represented by a quadruple $(P, Q, R, S)$ such that

(a) $S$ has no zeros in $D$;

(b) we can write

$$
\frac{P S-Q R}{S^{2}}=B U,
$$

where $B$ is the Blaschke product assigned to the problem (EI) in $\S 2$ and $U$ is a holomorphic function with $0<|U| \leq 1$ in $D$. For any $z_{0} \in D$ and $w_{0} \in D$ we may require moreover the condition

(c) $R\left(z_{0}\right) / S\left(z_{0}\right)=w_{0}$.

Proof. $1^{\circ}$. Let us prove the first statement of the theorem in the case where $\sigma=\left\{z_{1}, \ldots, z_{k}\right\}$ is finite, by induction on the total order $n=n_{1}+\cdots+n_{k}$. 
Suppose $n=1$. The equality

$$
\frac{f(z)-c_{10}}{1-\overline{c_{10}} f(z)}=\frac{z-z_{1}}{1-\bar{z}_{1} z} \cdot g(z)
$$

is equivalent to

$$
f(z)=\frac{P(z) g(z)+Q(z)}{R(z) g(z)+S(z)}
$$

where

$$
\left(\begin{array}{ll}
P(z) & Q(z) \\
R(z) & S(z)
\end{array}\right)=\left(\begin{array}{cc}
z-z_{1} & c_{10}\left(1-\overline{z_{1}} z\right) \\
\overline{c_{10}}\left(z-z_{1}\right) & 1-\bar{z}_{1} z
\end{array}\right)
$$

It is easy to see that $f \in \mathscr{E}$ if and only if $g \in \mathscr{B}$. Clearly $S(z) \neq 0$ in $D$. We have

$$
\frac{P S-Q R}{S^{2}}=\left(1-\left|c_{10}\right|^{2}\right) \cdot \frac{z-z_{1}}{1-\bar{z}_{1} z}=B U .
$$

By (H), one sees $\left|c_{10}\right|<1$ and $|U|=1-\left|c_{10}\right|^{2}$, so that (b) holds.

Suppose $n>1$ and that the first statement is valid for the problem of total order $<n$. Consider the transformation

$$
\frac{f(z)-c_{10}}{1-\overline{c_{10}} f(z)}=\frac{z-z_{1}}{1-\bar{z}_{1} z} \cdot \hat{f}(z),
$$

and, corresponding to $\hat{f}$, the following extended interpolation problem of total order $n-1$ :

$$
(\mathrm{EI})^{\wedge} \quad \hat{f}(z)=\sum_{\alpha=0}^{n_{i}-1} \hat{c}_{i \alpha}\left(z-z_{i}\right)^{\alpha}+\mathbf{O}\left(\left(z-z_{i}\right)^{\hat{n}_{i}}\right) \quad(i=1, \ldots, k),
$$

where $\hat{n}_{1}=n_{1}-1$ (if $\hat{n}_{1}=0$, there is no condition for $i=1$ ), $\hat{n}_{i}=n_{i}$ for $i=2, \ldots, k$, and the coefficients $\hat{c}_{i \alpha}\left(\alpha=0, \ldots, \hat{n}_{i}-1\right)$ are defined with the notations in $\S 2$ by

$$
\left(\begin{array}{cccc}
0 & & & \\
\hat{c}_{10} & \ddots & \\
\vdots & \ddots & & \\
\hat{c}_{1 \hat{n}_{1}-1} & \cdots & \hat{c}_{10} & 0
\end{array}\right)=\Delta\left(\left(1-\bar{z}_{1} z\right) \frac{f(z)-c_{10}}{1-\overline{c_{10}} f(z)} ; z_{1} ; n_{1}\right) ;
$$

and, for $i=2, \ldots, k$,

$$
\left(\begin{array}{ccc}
\hat{c}_{i 0} & & \\
\vdots & \ddots & \\
\hat{c}_{i \hat{n}_{1}-1} & \cdots & \hat{c}_{i 0}
\end{array}\right)=\Delta\left(\frac{1-\bar{z}_{1} z}{z-z_{1}} \frac{f(z)-c_{10}}{1-\overline{c_{10}} f(z)} ; z_{i} ; n_{i}\right) \text {. }
$$


Notice that, in this definition, it is enough to suppose that $f$ is holomorphic in the neighborhood of the set $\left\{z_{1}, \ldots, z_{k}\right\}$ and satisfies (EI). Recalling the formulas in $\S 2$ and rewriting (1) at $z_{1}$ in the form

$$
\left(1-\bar{z}_{1} z\right) \frac{f(z)-c_{10}}{1-\overline{c_{10}} f(z)}=\left(z-z_{1}\right) \cdot \sum_{\alpha=0}^{\hat{n}_{1}-1} \hat{c}_{1 \alpha}\left(z-z_{1}\right)^{\alpha}+\cdots,
$$

we see that $f$ is solution of (EI) in $\mathscr{B}$ if and only if $\hat{f}$ is solution of $(\mathrm{EI})^{\wedge}$ in $\mathscr{B}$. The relation (1) is equivalent to

$$
f(z)=\frac{\left(z-z_{1}\right) \hat{f}(z)+c_{10}\left(1-\bar{z}_{1} z\right)}{\overline{c_{10}}\left(z-z_{1}\right) \hat{f}(z)+\left(1-\bar{z}_{1} z\right)} .
$$

Evidently, the problem $(\mathrm{EI})^{\wedge}$ satisfies the hypothesis $(\mathrm{H})$ as well as the problem (EI).

By the hypothesis of induction, choose a parametrization for the problem $(\mathrm{EI})^{\wedge}$ represented by a quadruple $(\widehat{P}, \widehat{Q}, \widehat{R}, \widehat{S})$ satisfying (a) and (b). Put

$$
\left(\begin{array}{ll}
P(z) & Q(z) \\
R(z) & S(z)
\end{array}\right)=\left(\begin{array}{cc}
z-z_{1} & c_{10}\left(1-\bar{z}_{1} z\right) \\
c_{10}\left(z-z_{1}\right) & 1-\bar{z}_{1} z
\end{array}\right)\left(\begin{array}{cc}
\widehat{P}(z) & \widehat{Q}(z) \\
\widehat{R}(z) & \widehat{S}(z)
\end{array}\right)
$$

The function

$$
S(z)=\left(1-\bar{z}_{1} z\right) \widehat{S}(z)\left(\frac{z-z_{1}}{c_{10}} \frac{\widehat{Q}(z)}{1-\bar{z}_{1} z}+1\right)
$$

has no zeros in $D$, because $\left|c_{10}\right|<1$ by $(\mathrm{H})$ and $|\widehat{Q}|<|\widehat{S}|$ in $D$ by Proposition 3. From (3) one derives directly

$$
P S-Q R=\left(1-\left|c_{10}\right|^{2}\right)\left(z-z_{1}\right)\left(1-\bar{z}_{1} z\right)(\widehat{P} \widehat{S}-\widehat{Q} \widehat{R}) .
$$

Taking account of the Blaschke product assigned to $(\mathrm{EI})^{\wedge}$, we can write $(P S-Q R) / S^{2}=B U$, where $U$ is holomorphic and has no zeros in $D$. Since, for any $z_{0} \in D$, the radius

$$
\rho\left(z_{0}\right)=\left|P\left(z_{0}\right) S\left(z_{0}\right)-Q\left(z_{0}\right) R\left(z_{0}\right)\right| /\left(\left|S\left(z_{0}\right)\right|^{2}-\left|r\left(z_{0}\right)\right|^{2}\right)
$$

of disc $W\left(z_{0}\right)$ is smaller than 1 by Proposition 2, we have $|B U|<1$ and hence $0<|U| \leq 1$ in $D$. Thus (b) holds. Finally, a function $\hat{f}$ is solution of $(\mathrm{EI})^{\wedge}$ in $\mathscr{B}$ if and only if $\hat{f}$ is of the form

$$
\hat{f}=\frac{\widehat{P} g+\widehat{Q}}{\widehat{R} g+\widehat{S}} \quad \text { for some } g \in \mathscr{B} .
$$

This assertion and the relations (2) and (3) show that $(P, Q, R, S)$ represents a Nevanlinna parametrization of $\mathscr{E}$. 
$2^{\circ}$. Let us show that the first statement yields the second. Let $\left(P_{0}, Q_{0}, R_{0}, 1\right)$ represent a $\pi_{0} \in \mathscr{P}$ and take a $\tau \in G$ of the form

$$
\tau(z)=\lambda \frac{z+a}{1+\bar{a} z} \quad(|\lambda|=1,|a|<1) .
$$

Put

$$
\left(\begin{array}{ll}
P & Q \\
R & S
\end{array}\right)=\left(\begin{array}{cc}
P_{0} & Q_{0} \\
R_{0} & 1
\end{array}\right)\left(\begin{array}{cc}
\lambda & \lambda a \\
\bar{a} & 1
\end{array}\right)
$$

Then $(P, Q, R, S)$ represents $\tau^{*}\left(\pi_{0}\right)$. We can choose $\lambda$ and $a$ so that

$$
\frac{R\left(z_{0}\right)}{S\left(z_{0}\right)}=\lambda \frac{R_{0}\left(z_{0}\right)+\overline{\lambda a}}{1+\lambda a R_{0}\left(z_{0}\right)}=w_{0},
$$

because $\left|R_{0}\left(z_{0}\right)\right|<1$.

$3^{\circ}$. We proceed to prove the first statement in the case in which $\sigma$ is infinite. To each finite section $\sigma_{k}=\left\{z_{1}, \ldots, z_{k}\right\}$ of $\sigma \quad(k=$ $1,2, \ldots)$, we associate the following extended interpolation problem:

$$
(\mathrm{EI})_{k} \quad f(z)=\sum_{\alpha=0}^{n_{i}-1} c_{i \alpha}\left(z-z_{i}\right)^{\alpha}+\mathbf{O}\left(\left(z-z_{i}\right)^{n}\right) \quad(i=1, \ldots, k) .
$$

Let $\mathscr{E}_{k}$ denote the set of all solutions of $(\mathrm{EI})_{k}$ in $\mathscr{B}$. Clearly $\mathscr{E}=$ $\bigcap_{k \in \mathbb{N}} \mathscr{E}_{k}$. By assumption, $\mathscr{E}_{k}$ has at least two elements.

Fix a point $z_{0} \in D$ and take, for each $k$, by $1^{\circ}$ and $2^{\circ}$, a Nevanlinna parametrization of $\mathscr{E}_{k}$ represented by $\left(P_{k}, Q_{k}, R_{k}, 1\right)$ such that the condition (b) holds and $R_{k}\left(z_{0}\right)=0$. By Proposition 3, we can choose a subsequence $\left\{k_{j}\right\}$ of $\mathbb{N}$ such that $\left\{P_{k_{j}}\right\},\left\{Q_{k_{j}}\right\}$, and $\left\{R_{k}\right\}$ converge to holomorphic functions $P, Q$, and $R$ in $D$ respectively. We have $R \in \mathscr{B}$ and $R\left(z_{0}\right)=0$, and hence $|R|<1$ and $R g+1 \neq 0$ in $D$ for any $g \in \mathscr{B}$. A normal families argument shows immediately that $(P, Q, R, 1)$ represents a parametrization of $\mathscr{E}$.

It remains to verify (b). Write for each $k$

$$
P_{k}-Q_{k} \cdot R_{k}=B_{k} \cdot U_{k},
$$

where $B_{k}$ is the Blaschke product assigned to $(\mathrm{EI})_{k}$ and $U_{k}$ is holomorphic function with $0<\left|U_{k}\right| \leq 1$ in $D$. The subsequence $\left\{U_{k_{j}}\right\}$ converges evidently to a function $U \in \mathscr{B}$. If $U \equiv 0$ in $D$, then $P-Q R \equiv 0$ and all solutions in $\mathscr{E}$ would be reduced to a same constant, contrary to $(\mathrm{H})$. Thus $0<|U| \leq 1$. Since $\left\{B_{k_{j}}\right\}$ converges to $B$, this completes the proof of Theorem 2 . 
By (b) of Theorem 2 and the note at the end of $\S 3$, we have

Corollary 1. Under the hypothesis $(\mathrm{H})$, let $\pi \in \mathscr{P}$ and $z_{0} \in D \backslash \sigma$. Then $\pi_{z_{0}}: \bar{D} \rightarrow W\left(z_{0}\right)$ is bijective and $W\left(z_{0}\right)$ is a nondegenerate closed disc contained in $D$. If $(P, Q, R, S)$ represents $\pi$ then the center and the radius of $W\left(z_{0}\right)$ are

$$
\left(Q\left(z_{0}\right) \overline{S\left(z_{0}\right)}-P\left(z_{0}\right) \overline{R\left(z_{0}\right)}\right) /\left(\left|S\left(z_{0}\right)\right|^{2}-\left|R\left(z_{0}\right)\right|^{2}\right)
$$

and

$$
\left|P\left(z_{0}\right) S\left(z_{0}\right)-Q\left(z_{0}\right) R\left(z_{0}\right)\right| /\left(\left|S\left(z_{0}\right)\right|^{2}-\left|R\left(z_{0}\right)\right|^{2}\right)
$$

respectively.

Corollary 2. Under the hypothesis $(\mathrm{H})$, let $\pi \in \mathscr{P}$. If $(P, Q$, $R, S)$ represents $\pi$ then we can write

$$
\frac{P S-Q R}{S^{2}}=B U
$$

where $U$ is a holomorphic function with $0<|U| \leq 1$ in $D$.

To see Corollary 2 , let $\pi_{0} \in \mathscr{P}$ be represented by a $\left(P_{0}, Q_{0}, R_{0}, S_{0}\right)$ satisfying the conditions (a) and (b) of Theorem 2. By Theorem 1, there exists $\tau \in G$ such that $\pi=\tau^{*}\left(\pi_{0}\right)$. Write

$$
\tau(z)=\frac{p z+q}{\bar{q} z+\bar{p}} \quad\left(|p|^{2}-|q|^{2}=1\right) .
$$

Put

$$
\left(\begin{array}{ll}
P^{\prime} & Q^{\prime} \\
R^{\prime} & S^{\prime}
\end{array}\right)=\left(\begin{array}{ll}
P_{0} & Q_{0} \\
R_{0} & S_{0}
\end{array}\right)\left(\begin{array}{ll}
p & q \\
\bar{q} & \bar{p}
\end{array}\right)
$$

Then $\left(P^{\prime}, Q^{\prime}, R^{\prime}, S^{\prime}\right)$ represents $\pi$ also and we have

$$
\frac{P S-Q R}{S^{2}}=\frac{P^{\prime} S^{\prime}-Q^{\prime} R^{\prime}}{S^{\prime 2}}=\left(\frac{S_{0}}{S^{\prime}}\right)^{2} \frac{P_{0} S_{0}-Q_{0} R_{0}}{S_{0}^{2}} .
$$

Since $\left|S_{0}\right|>\left|R_{0}\right|$ in $D$ and $|p|>|q|, S^{\prime}$ has no zeros in $D$. Thus we can write $(P S-Q R) / S^{2}=B U$, where $U$ is holomorphic and has no zeros in $D$. For each $z_{0} \in D$, the radius of $W\left(z_{0}\right)$ is smaller than 1 , so that we have $|B U|<1$ in $D$. The well-known theorem on the factorization of bounded analytic functions yields $0<|U| \leq 1$ in $D$.

Notes. (i) In Theorem 1 , we see by $1^{\circ}$ of its proof that, in the finite case, we may require moreover the fourth condition

(d) $P, Q, R$, and $S$ are polynomials in $z$ of degree $\leq n$. 
(ii) In the finite case, by (1) in $1^{\circ}$ and from the fact that if $f$ is a finite Blaschke product of degree $m$ then $\tau \circ f$ is also such that for any $\tau \in G$, we see by induction that, for any $\pi \in \mathscr{P}$ and $\zeta \in \partial D$, $\pi(\zeta)$ is a Blaschke product of degree $n$.

(iii) In the infinite case, the argument of Nevanlinna used in [13] shows that, for any $\pi \in \mathscr{P}$ and $\zeta \in \partial D, \pi(\zeta)$ is an inner function.

6. Discs $W^{\prime}\left(z_{i}\right)$. In this section, we study the closed discs

$$
W^{\prime}\left(z_{i}\right)=\left\{f^{\left(n_{t}\right)}\left(z_{i}\right): f \in \mathscr{E}\right\}
$$

by means of a Nevanlinna parametrization. Let $\pi \in \mathscr{P}$ be represented by $(P, Q, R, S)$ such that $S \neq 0$ in $D$. For the sake of simplicity, for each $z_{i} \in \sigma$ and $f \in \mathscr{B}$, we shall denote the value of the $n_{i}$ th derivative of $f$ at $z_{i}$ by

$$
d_{i} f=f^{\left(n_{i}\right)}\left(z_{i}\right) \text {. }
$$

The following lemma shows that, for $g \in \mathscr{B}, d_{i}(\pi(g))$ depends only on $\pi$ and the value of $g$ at $z_{i}$.

Lemma 3. Let $g, g_{0} \in \mathscr{B}$. For any $z_{i} \in \sigma$ we have

$$
d_{i}(\pi(g))-d_{i}\left(\pi\left(g_{0}\right)\right)=\frac{\left(g\left(z_{i}\right)-g_{0}\left(z_{i}\right)\right) \cdot d_{i}(P S-Q R)}{\left(R\left(z_{i}\right) g\left(z_{i}\right)+S\left(z_{i}\right)\right)\left(R\left(z_{i}\right) g_{0}\left(z_{i}\right)+S\left(z_{i}\right)\right)} .
$$

In fact, a simple calculation yields

$$
\pi(g)-\pi\left(g_{0}\right)=\frac{g-g_{0}}{(R g+S)\left(R g_{0}+S\right)}(P S-Q R) .
$$

Since $|S|>|R|$ and $P S-Q R$ vanishes at $z_{i}$ with the order $n_{i}$, we have at once the relation of the lemma.

THEOREM 3. Under the hypothesis $(\mathbf{H})$, let $\pi$ be a Nevanlinna parametrization of $\mathscr{E}$ represented by a quadruple $(P, Q, R, S)$ such that $S$ has no zeros in $D$. Then we have, for each $z_{i} \in \sigma$, with the notation $d_{i}$ defined above,

$$
W^{\prime}\left(z_{i}\right)=\left\{d_{i}(Q / S)+\frac{d_{i}(P S-Q R) \cdot \zeta}{\left(R\left(z_{i}\right) \zeta+S\left(z_{i}\right)\right) S\left(z_{i}\right)}: \zeta \in \bar{D}\right\} .
$$

Thus, $W^{\prime}\left(z_{i}\right)$ is a nondegenerate compact disc of radius

$$
\frac{\left|d_{i}(P S-Q R)\right|}{\left|S\left(z_{i}\right)\right|^{2}-\left|R\left(z_{i}\right)\right|^{2}} \text {. }
$$

To see this, it is enough to put $g_{0} \equiv 0$ in Lemma 3 and recall Proposition 2. 
Note that in the case where $\sigma$ is finite we showed in our recent report [25] that $W^{\prime}\left(z_{i}\right)$ is a nondegenerate compact disc without using Nevanlinna parametrizations.

From Lemma 1 in $\S 3$, Corollary 1 in $\S 5$, and the above expression of $W^{\prime}\left(z_{i}\right)$ of Theorem 3, we sum up the following properties of the extremal solutions of $(\mathrm{EI})$ in $\mathscr{B}$.

Corollary 3. Under the hypothesis $(\mathrm{H})$, let $\pi \in \mathscr{P}$ and $f \in \mathscr{E}$. The following conditions are equivalent:

(a) $f\left(z_{0}\right) \in \partial W\left(z_{0}\right)$ for some $z_{0} \in D \backslash \sigma$.

(b) $f(z) \in \partial W(z)$ for any $z \in D$.

(c) $f^{\left(n_{i}\right)}\left(z_{i}\right) \in \partial W^{\prime}\left(z_{i}\right)$ for some $z_{i} \in \sigma$.

(d) $f^{\left(n_{i}\right)}\left(z_{i}\right) \in \partial W^{\prime}\left(z_{i}\right)$ for any $z_{i} \in \sigma$.

(e) There exists a $\zeta \in \partial D$ such that $f=\pi(\zeta)$.

If one of (and hence all of) these conditions is satisfied, $\zeta$ in (e) is uniquely determined by $\pi$ and $f$.

7. Remarks on the existence and the uniqueness of solutions. In this section, we state some remarks on the existence and the uniqueness of solutions of $(E I)$ in $\mathscr{B}$, in the case where the sequence $\sigma$ is infinite.

As in $\S 5$, for each finite section $\sigma_{k}=\left\{z_{1}, \ldots, z_{k}\right\}$ of $\sigma \quad(k=$ $1,2, \ldots)$, we consider the problem $(\mathrm{EI})_{k}$, and the set $\mathscr{E}_{k}$ of solutions of $(\mathrm{EI})_{k}$ in $\mathscr{B}$. Clearly $\mathscr{E}_{k} \supset \mathscr{E}_{k+1}$ and $\mathscr{E}=\bigcap_{k \in B \mathbb{N}} \mathscr{E}_{k}$. Let $A_{k}$ be the Hermitian matrix of $(\mathrm{EI})_{k}$, mentioned in the Introduction and defined in [24].

A normal families argument shows immediately that there exists a solution of (EI) in $\mathscr{B}$, that is, $\mathscr{E} \neq \varnothing$, if and only if $A_{k} \geq 0$, that is, $\mathscr{E}_{k} \neq \varnothing$, for all $k \in \mathbb{N}$.

Assuming $\mathscr{E} \neq \varnothing$, we proceed to the uniqueness. We distinguish two cases:

Case I where $\operatorname{det} A_{k}=0$ for some $k$;

Case II where $\operatorname{det} A_{k}>0$ for all $k$.

In Case I, by Theorem $\mathrm{U}$ in the Introduction, the solution of (EI) in $\mathscr{B}$ is evidently unique and it is a Blaschke product of degree < $n_{1}+\cdots+n_{k}$. In Case II, each $(\mathrm{EI})_{k}$ has an infinite number of solutions in $\mathscr{B}$. But it happens that the solution of $(E I)$ in $\mathscr{B}$ may be unique. From now on we restrict ourselves to the Case II.

For any $z_{0} \in D$, let $W_{k}\left(z_{0}\right)=\left\{f\left(z_{0}\right): f \in \mathscr{E}_{k}\right\}$. By Corollary 1, $W_{k}\left(z_{0}\right)$ is a nondegenerate closed disc in $D$, provided that $z_{0} \notin \sigma_{k}$. 
We easily see $W_{k}\left(z_{0}\right) \supset W_{k+1}\left(z_{0}\right)$ and $W\left(z_{0}\right)=\bigcap_{k \in \mathbb{N}} W_{k}\left(z_{0}\right)$. The radius $\rho_{k}\left(z_{0}\right)$ of $W_{k}\left(z_{0}\right)$ converges to the radius $\rho\left(z_{0}\right)$ of $W\left(z_{0}\right)$ as $k \rightarrow \infty$.

By Corollary 1 , we observe at once that if $\rho_{k}\left(z_{0}\right) \rightarrow 0($ as $k \rightarrow \infty)$ for some $z_{0} \in D \backslash \sigma$ then $\rho_{k}(z) \rightarrow 0$ for all $z \in D$ and hence the solution of $(\mathrm{EI})$ in $\mathscr{B}$ is unique.

Finally, we note that, by means of Schur-Nevanlinna's algorithm [13], we obtain Denjoy's criterion of uniqueness [4], in our extended case too. This criterion involves unhappily a sequence of constants that we cannot determine directly in terms of the given data $\left\{z_{i}\right\}$ and $\left\{c_{i \alpha}\right\}$. Here we are content to indicate only the first step of the algorithm.

Fix a point $z_{0} \in D \backslash \sigma$ and consider the following transformation

$$
\frac{f_{1}-c_{10}}{1-\overline{c_{10}} f_{1}}=\frac{z-z_{1}}{1-\bar{z}_{1} z} \cdot \frac{f_{2}-a}{1-\bar{a} f_{2}}
$$

with the constant $a=c_{10}\left(\bar{z}_{0}-\bar{z}_{1}\right) /\left(1-z_{1} \bar{z}_{0}\right)$. As in $\S 5$, we see that $f_{1} \in \mathscr{E}$ (resp $f_{1} \in \mathscr{E}_{k}$ ) if and only if $f_{2}$ is a solution in $\mathscr{B}$ of another extended interpolation problem $(\mathrm{EI})^{(2)}$ (resp $\left.(\mathrm{EI})_{k}^{(2)}\right)$ defined from (EI) by means of (4), analogously to $(\mathrm{EI})^{\wedge}$ in $\S 5$. The order $n_{i}^{(2)}$ of $(\mathrm{EI})^{(2)}$ at $z_{i}$ is equal to $n_{1}-1$ for $i=1$ and to $n_{i}$ for $i \geq 2$. Put $z^{(1)}=z_{1}, c^{(1)}=c_{10} ; z^{(2)}=z_{2}$ if $n_{1}=1$, and $z^{(2)}=z_{1}$ if $n_{1} \geq 2$; and $c^{(2)}=f_{2}\left(z^{(2)}\right)$ for some solution $f_{2}$ of $(\mathrm{EI})^{(2)}$. Rewrite (4) in the form

$$
f_{1}=\frac{P f_{2}+Q}{R f_{2}+1}
$$

with

$$
u=\frac{z-z_{1}}{1-\bar{z}_{1} z}, \quad P=\frac{u-c_{10} \bar{a}}{1-\overline{c_{10}} a u}, \quad Q=\frac{c_{10}-a u}{1-\overline{c_{10}} a u}, \quad R=\frac{\overline{c_{10}} u-\bar{a}}{1-\overline{c_{10}} a u} .
$$

By the definition of $a$, we have $R\left(z_{0}\right)=0$. Put

$$
\eta^{(1)}=\left|P\left(z_{0}\right)\right|=\left|u^{(1)}\right| \frac{1-\left|c^{(1)}\right|^{2}}{1-\left|c^{(1)}\right|^{2}\left|u^{(1)}\right|^{2}},
$$

where $\left|u^{(1)}\right|=\left|z_{0}-z^{(1)}\right| /\left|1-\overline{z^{(1)}} z_{0}\right|$ is the noneuclidean pseudodistance between $z_{0}$ and $z^{(1)}$. The relation (5) shows that $\eta^{(1)}$ is the ratio of the radius of $W_{k}\left(z_{0}\right)$ to that of $W_{k}^{(2)}\left(z_{0}\right)=\left\{f_{2}\left(z_{0}\right): f_{2}\right.$ is solution of $(E I)_{k}^{(2)}$ in $\left.\mathscr{B}\right\}$; and so on. Thus,

$$
\rho_{k}\left(z_{0}\right)=\prod_{j=1}^{n_{1}+\cdots+n_{k}} \eta^{(j)}
$$


Denjoy's criterion in our case says that the uniqueness is equivalent to the divergence of the infinite product $\prod_{j \in \mathbb{N}} \eta^{(j)}$ or the divergence of

$$
\sum_{j \in \mathbb{N}} \frac{1-\left|u^{(j)}\right|}{1-\left|c^{(j)}\right|}
$$

Acknowledgment. The author is grateful to the referee for suggesting the revision of this paper and drawing her attention to the reference [10].

\section{REFERENCES}

[1] V. M. Adamyan, D. Z. Arov and M. G. Krein, Infinite Hankel matrices and generalized problem of Carathéodory-Fejér and I. Schur, Functional Anal. Appl., 2 (1968), 269-281.

[2] L. Ahlfors, Conformal Invariants. Topics in Geometric Function Theory, McGraw-Hill, New York, 1973.

[3] C. Carathéodory, Über den Variabilitätsbereich der Fourier'schen Konstanten von positiven harmonischen Funktionen, Rend. Circ. Mat. Palermo, 32 (1911), 193-217.

[4] A. Denjoy, Sur une classe de fonctions analytiques, C. R. Acad. Sci. (Paris), 188 (1929), 140-142; 1084-1086.

[5] A. M. Dzrbašjan, Multiple interpolation in the $H^{p}$ classes, $0<p \leq \infty$, Soviet Math. Dokl., 18 (1977) 837-841.

[6] J. P. Earl, On the interpolation of bounded sequences by bounded functions, J. London Math. Soc. (2), 2 (1970), 544-548.

[7] _ A note on bounded interpolation in the unit disc, J. London. Math. Soc. (2), 13 (1976), 419-423.

[8] J. B. Garnett, Two remarks on interpolation by bounded analytic functions. Lect. Notes in Math., 604 (1977).

[9] _ Bounded Analytic Functions, Academic Press, New York, 1981.

[10] D. M. Koteliansky, Sur certaines applications des formes quadratiques au problème de Nevanlinna-Pick, J. Inst. Math. Acad. Sci. Ukraine Nr 1, 73-87 u. franz. Zusammenfassung 87-88 (1937).

[11] D. E. Marshall, An elementary proof of Pick-Nevanlinna interpolation theorem, Michigan Math. J., 21 (1974), 219-223.

[12] V. M. Martirosyan, Effective solution of an interpolation problem with nodes of bounded multiplicity, Soviet Math. Dokl., 25 (1982), 441-445.

[13] R. Nevanlinna, Über beschränkte analytische Funktionen, Ann. Acad. Sci. Fenn. Ser A, 32 (1929), No. 7.

[14] K. Øyma, Extremal interpolatory functions in $H^{\infty}$, Proc. Amer. Math. Soc., 64 (1977), 272-276.

[15] _ Interpolation in $H^{p}$-spaces, Proc. Amer. Math. Soc., 76 (1979), 81-88.

[16] _- An interpolation theorem for $H_{E}^{\infty}$, Pacific J. Math., 109 (1983), 457-462.

[17] G. Pick, Über die Beschränkungen analytischer Funktionen, welche durch vorgegebene Funktionswerte bewirkt werden, Math. Ann., 77 (1915), 7-23.

[18] A. M. Russakovskii, The problem of multiple interpolation in the class of functions that are analytic in a half-plane and hence indicators not exceeding a given one, Soviet Math. Dokl., 27 (1983), 433-436. 
[19] I. Schur, Über Potenzreihen, die im Innern des Einheitskreises beschränkt sind, J. Riene Angew. Math., 147 (1917), 205-232.

[20] A. Stray, Two applications of the Schur-Nevanlinna algorithm, Pacific J. Math., 91 (1980), 223-232.

[21] _- On a formula by V. M. Adamjan, D. Z. Arov and M. G. Krein, Proc. Amer. Math. Soc., 83 (1981), 337-340.

[22] _ Minimal interpolation by Blaschke products, J. London Math. Soc. (2), 32 (1985), 488-496.

[23] A. Stray and K. O. Øyma, On interpolations with minimal norm, Proc. Amer. Math. Soc., 68 (1978), 75-78.

[24] S. Takahashi, Extension of the theorems of Carathéodory-Toeplitz-Schur and Pick, Pacific J. Math., 138 (1989), 391-399.

[25] __ Extended interpolation problems for bounded analytic functions, Annual Reports of Graduate School of Human Culture (Nara Women's University), 4 (1989), 107-119.

[26] O. Toeplitz, Über die Fouriers'che Entwicklung positiver Funktionen, Rend. Circ. Math. Palermo, 32 (1911), 191-192.

Received November 18, 1988 and in revised form August 1, 1989.

NARA WOMEN'S UNIVERSITY

NARA, 630 JAPAN 



\section{PACIFIC JOURNAL OF MATHEMATICS EDITORS}

\author{
V. S. VARADARAJAN \\ (Managing Editor) \\ University of California \\ Los Angeles, CA 90024-1555-05 \\ Herbert Clemens \\ University of Utah \\ Salt Lake City, UT 84112 \\ Thomas ENRIGHT \\ University of California, San Diego \\ La Jolla, CA 92093
}

\section{R. FINN}

Stanford University

Stanford, CA 94305

Hermann FlaschKa

University of Arizona

Tucson, AZ 85721

VAUGHAN F. R. Jones

University of California

Berkeley, CA 94720

STEVEN KeRCKHOFF

Stanford University

Stanford, CA 94305
C. C. MOORE

University of California

Berkeley, CA 94720

Martin ScharlemanN

University of California

Santa Barbara, CA 93106

HAROLd STARK

University of California, San Diego

La Jolla, CA 92093

\section{ASSOCIATE EDITORS}
R. ARENS
E. F. BECKENBACH
B. H. NeumanN
F. Wolf
K. YoshidA (1906-1982)
(1904-1989)

\section{SUPPORTING INSTITUTIONS}
UNIVERSITY OF ARIZONA
UNIVERSITY OF BRITISH COLUMBIA
CALIFORNIA INSTITUTE OF TECHNOLOGY
UNIVERSITY OF CALIFORNIA
MONTANA STATE UNIVERSITY
UNIVERSITY OF NEVADA, RENO
NEW MEXICO STATE UNIVERSITY
OREGON STATE UNIVERSITY
UNIVERSITY OF OREGON
UNIVERSITY OF SOUTHERN CALIFORNIA
STANFORD UNIVERSITY
UNIVERSITY OF HAWAII
UNIVERSITY OF TOKYO
UNIVERSITY OF UTAH
WASHINGTON STATE UNIVERSITY
UNIVERSITY OF WASHINGTON 


\section{Pacific Journal of Mathematics}

Vol. 146, No. $1 \quad$ November, 1990

Primo Brandi and Anna Salvadori, A quasi-additivity type condition and

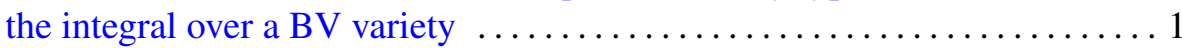

Dong M. Chung, Chull Park and David Lee Skoug, Operator-valued

Feynman integrals via conditional Feynman integrals ..............21

Paul Jolissaint, Index for pairs of finite von Neumann algebras . .........43

Miodrag Mateljević and Miroslav Pavlović, Multipliers of $H^{p}$ and

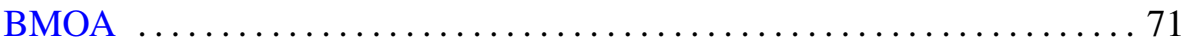

Himadri Kumar Mukerjee, Poincaré cobordism exact sequences and

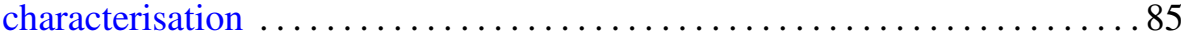

Thomas H. Otway, The coupled Yang-Mills-Dirac equations for differential

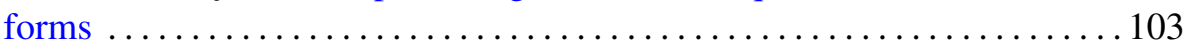

Sechiko Takahashi, Nevanlinna parametrizations for the extended

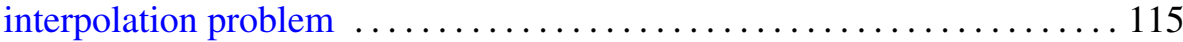

P. C. Trombi, Uniform asymptotics for real reductive Lie groups ........ 131 